Trotz der sozialen Unzulänglichkeiten treffen wir hier lebensfrohe Menschen, mit orientalischem Einschlag, Mediterranerkultur und katholischer Tradition. In den Dörfern werden den Sommer über Festas, die Namenstage der Schutzheiligen, gefeiert und in der Stadt die allgemeinen Feste Karneval, Sonnenwende, St. Peter und Paul, Busket-Gardèn mit Sing- und Tanzgruppen, am 8. September der Nationaltag (Siège), der Gedenktag des Sieges über die Türken 1565 und des Widerstandes im 2. Weltkrieg. Flächenmäßig sind die Malteser In eln begrenzt, aber sie sind reich an archäologischen Zeugen und an historischen Bauwerken.

Das Jahr 1964 ist für Malta von außerordentlicher Bedeutung, da die Inseln im Frühjahr die volle Unabhängigkeit von Großbritannien erlangen werden. In volkswirtschaftlicher Hinsicht wird es noch nicht selbständig sein und zunächst auf fremde Hilfe angewiesen bleiben.

\title{
DAS HAUS DES DJERID
}

\author{
KARL SUTER
}

Unter dem Bled el Djerid, d.h. Land der Dattelpalme (djerid = Palmwedel), versteht man eine kleinere, sich westlich des Chott el Djerid ausdehnende Region Südtunesiens. Sie wird gewöhnlich einfach Djerid genannt. Sie weist einige Oasen auf, darunter als wichtigste Tozeur und Nefta, beide über Tunesien hinaus durch ihren Reichtum an artesisch aufsteigendem Grundwasser, durch ihre herrlichen Palmgärten und durch die Originalität ihrer Hausbauten bekannt. Diese Bauten haben, mit jenen anderer tunesischer Regionen verglichen, einen besondern Charakter. Das kommt schon im verwendeten Baumaterial, dem gebrannten Backstein (galeb, geleb), zum Ausdruck. Ton (tin), der sich für die Backsteinherstellung eignet, ist in reichlichen Mengen in der Nähe der Siedlungen vorhanden. Er wird, gut durchnäßt, mit den Füßen zu einem Brei geknetet. Mit diesem füllt man die zwei Fächer eines Holzrahmens von Hand aus, schlägt die beiden entstandenen Lehmziegel heraus und wiederholt diesen Vorgang so oft, bis der ganze Brei aufgebraucht ist. Das geht sehr rasch; in kurzer Zeit ist der Boden mit Dutzenden von Lehmziegeln (toub) bedeckt. Diese läßt man an Sonne und Luft trocknen, und zwar je nach der gerade herrschenden Temperatur während ein bis fünf Tagen. Dann kommen sie für 24-36 Stunden in den Ofen. Sie haben, einmal gebrannt, eine Länge von $25 \mathrm{~cm}$, eine Breite von $10 \mathrm{~cm}$ und eine Dicke von $4 \mathrm{~cm}$. Als Mörtel wird ein Gemisch aus Ton und Sand verwendet.

Die Grundmauern des Hauses erhalten die beträchtliche Dicke von $40-80 \mathrm{~cm}$. Sowohl nach der Gasse als auch nach dem Hof hin treten sie als reine Backsteinfronten entgegen, besitzen aber im Innern meistens eine Füllschicht aus kleinern Kalksteinen. Nicht indes die dünnern, das Hausinnere unterteilenden $Z$ wischenwände. Selbst wo ein Haus, was seit wenigen Jahren, wenn auch selten, vorkommt, ganz aus Kalkstein und Kalkmörtel gebaut wird, werden doch noch Backsteine benötigt, im besondern für den Mauerrahmen um die Tür- und Fensteröffnungen herum, denn dieser Stein eignet sich besser für schöne geradlinige Mauerabschlüsse als der Bruchstein.

Was den Gehöften (hausch, franz. haouch) eine besondere Note verleiht, ist die abwechslungsreiche Ornamentierung ihrer Fassaden, und zwar sowohl jener gegen die Gasse als auch gegen den Hof hin. Da sind dem Einfall und der Einbildungskraft keine Grenzen gesetzt. Wer diese Siedlungen durchwandert, stößt immer wieder auf neue Muster. Sie stellen gebrochene Linienzüge und einfachere geometrische Flächen und Figuren in mannigfacher Abwandlung dar. Besonders schöne Verzierungen weist oft die 


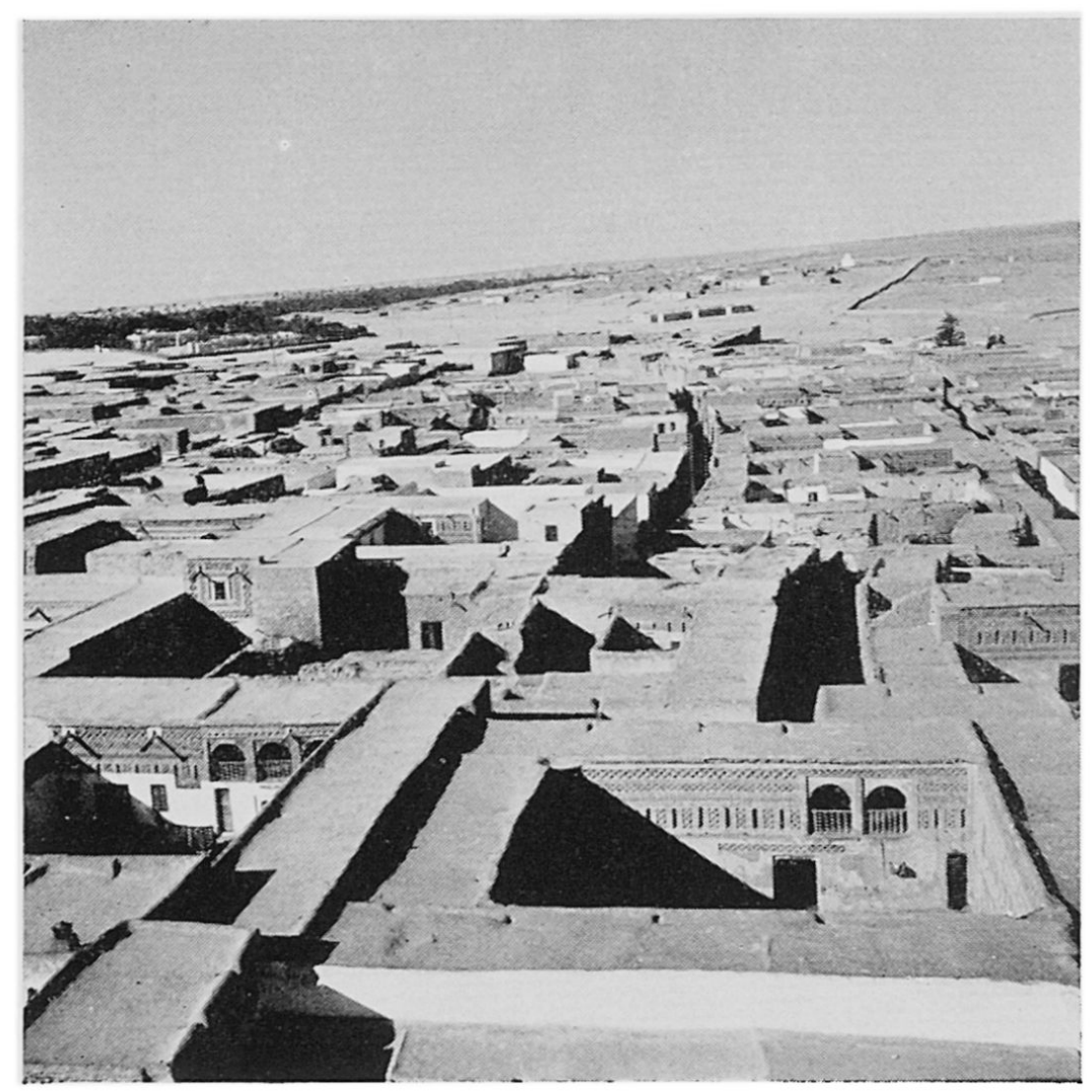

Abb. 1: Tozeur

Blick über einen Teil der Siedlung. Die Hausterrassen sind von einem niedrigen Mauerbord umzogen. Die Fassaden gegen den $\mathrm{Hof}$ hin sind von Mauerschlitzen durchsetzt, zeigen Arkaden und sind im unteren Teil oft geweißt.

Fensterwand des direkt über der Gasse gelegenen Gemachs auf. A. Borg ${ }^{1}$ betrachtet diese Backsteinornamentik als das originellste Merkmal des Hauses des Djerid (S. 90).

Die Decken bzw. Terrassen (stah) werden aus Palmbalken konstruiert. Dazu wird, wenn immer möglich, nur Holz der besten Palmsorten verwendet, namentlich das der unter dem Namen Besserhelou bekannten. Dieses Holz, das relativ wenig biegsam ist, erlaubt Mauerabstände und damit Zimmerbreiten bis $\mathrm{zu} 4 \mathrm{~m}$. Das ist viel, betragen die Abstände doch gewöhnlich, wie z. B. in zahlreichen Oasen der algerischen Sahara, nur 2,5 m. Doch gibt man sich in Tozeur und Nefta auch mit geringeren Abständen zufrieden, mit solchen von bloß 3-3,2 m. Der Stamm wird je nach Qualität in Teilstücke von 3,5-4,5 m Länge zerlegt und das einzelne Stück der Länge nach noch in zwei, seltener in drei weitere Teile. Aus diesen Balken bildet man über benachbarten Grundmauern einen Rost mit Balkenabständen von $0,15-0,35 \mathrm{~m}$ oder, falls man je zwei Balken geschlossen aneinanderfügt, von 1-1,2 m. Nun müssen noch die verbleibenden $Z$ wischenräume ausgefüllt werden. Das geschieht in der Regel gleichfalls mit Palmenholz, doch mit solchem von geringerer Qualität, gelegentlich benützt man dazu, besonders über den Ställen, auch bloß Palmwedel. Ein Balken kommt dicht neben den andern in der Verlaufsrichtung der Mauern über den Rost zu liegen. Nun hat noch die Abdichtung der Decke gegen Wind und Wetter zu erfolgen. Hiezu eignet sich Tonbrei, mit dem man das ganze Balkenwerk überzieht, und Gips- oder Kalkmörtel für den endgültigen Abschluß. Diese Decken vertragen aber kein Regenwasser. Darum werden in Abständen von 2-3 m etwa $1 \mathrm{~m}$ lange Ablaufrinnen aus ausgehöhlten Palmbalken, in neuerer Zeit auch aus ins Djerid eingeführten Blechrohren angebracht. Diese Dachrinnen sind gewöhnlich gegen den Innenhof gerichtet, gelegentlich aber auch gegen die

1 L'habitat rural au Maroc, en Algérie et en Tunisie. Darin A. Borg: L'habitat à Tozeur, S. 91-107 (mit zahlreichen Abbildungen), Cahiers des Arts et Techniques d'Afrique du Nord. Editions Privat, Toulouse, 1959. 


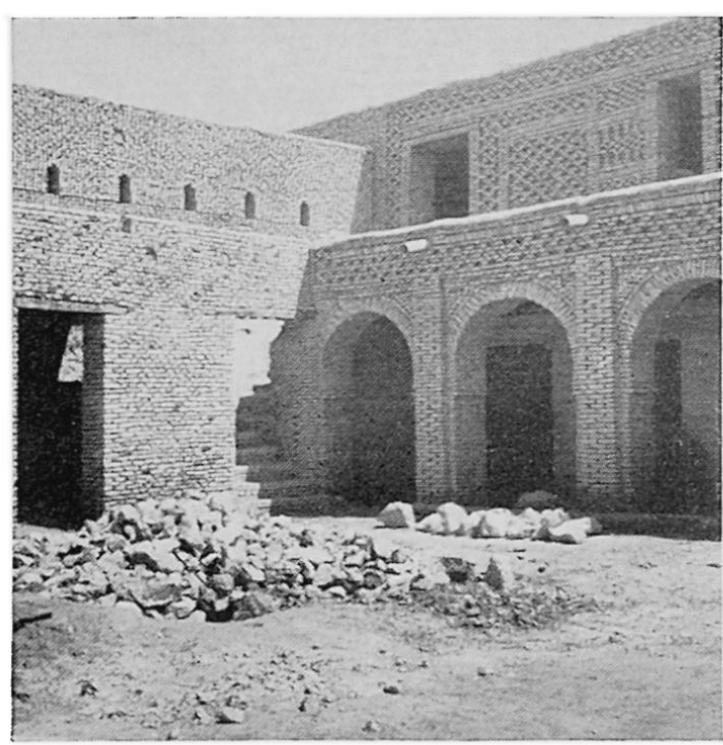

Abb. 2: Tozeur

Neuer Backsteinbau mit Arkaden auf einer Seite des Hofes, hinter der Wohn- und Schlafräume liegen. Eine Treppe führt zu einem Speicherraum im ersten Stock hinauf

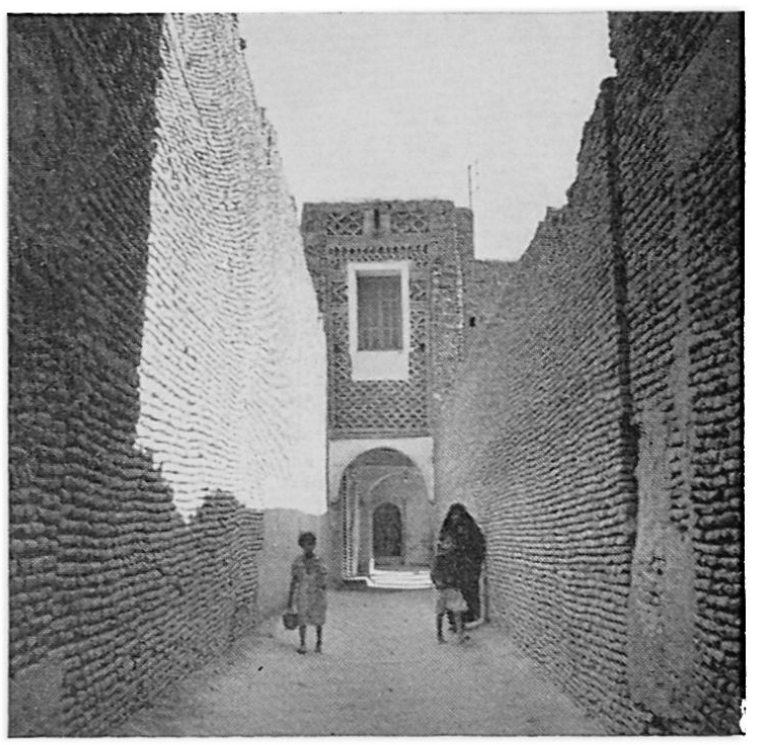

Abb. 3: Tozeur

Schmale, teils offene, teils infolge Überbauung gedeckte Gasse

Gasse, auf die dann bei starkem Regen ein wahrer Wasserschwall stürzt. Die Terrassen werden, um das Wasser am Abfließen über die Hausmauern zu verhindern, von einem niedrigen Mauerbord eingefaßt.

Die Gehöfte sind meist groß und, wenn auch in Einzelheiten voneinander abweichend, nach dem gleichen Grundplan gebaut. Ein Gang (skifa) führt, winklig gebogen, von der Gasse aus unter der Hausterrasse hindurch in den quadratischen oder rechteckigen Innenhof. Darum ist es selbst bei sperrangelweit offener Tür nicht möglich, in den Hof hinein zu sehen. Der vordere Abschnitt des Ganges wird als erste, der hintere als zweite Skifa bezeichnet und der dritte Abschnitt, falls überhaupt vorhanden, als Portal. Dem ersten Abschnitt kommt eine besondere Bedeutung für das Gemeinschaftsleben zu. Hier versammelt sich das Männervolk bei Hausfesten und hier pflegen die männlichen Hausinsaßen öfters mit Gästen zusammen 'Tee zu trinken. Darum ist dieser Abschnitt längs seiner Wände mit steinernen Sitzbänken (doukana) ausgestattet. In den Häusern der wohlhabenderen Familien dient diesem Gemeinschaftsleben ein besonderer, von der ersten Skifa aus erreichbarer Raum. Nur ausnahmsweise ist er mit dem Hof durch eine Tür direkt verbunden. In der zweiten Skifa pflegen, wie schon A. Borg (S.93) erwähnt, die Frauen während den heißen Sommermonaten zu weben, ist es doch selbst dann hier wegen ständigen Luftzugs verhältnismäßig kühl. Im Winter obliegen sie dieser Beschäftigung im Hof oder unter der in gewissen Häusern den Hof auf einer Seite begleitenden Arkade oder gar in einem besondern Webraum.

Um den Hof (uast el hausch $=$ das Innere des Hauses) gruppieren sich hauptsächlich Wohn/Schlafräume (dar, bit), in den kleinern Gehöften zwei oder drei, in den größeren fünf bis acht, und zwar je einer für die Eltern und für die einzelnen Sohnsfamilien. Denn die verheirateten Söhne leben mit den Eltern, eine Großfamilie bildend, zusammen. Diese Räume sind hoch, und zwar mindestens 3,5-4 m, im allgemeinen jedoch 6-10 m und weisen gegen den Hof hin Fenster auf, welche durch Läden aus eingeführtem Tannenholz verschließbar sind. Sie sind nach Europäerart möbliert, mit elektrischem Licht versehen und manchmal durch eine Querwand in einen Wohn- und Schlafabschnitt unterteilt; ihre Böden bestehen aus Fliesen. Häufig kommt es vor, daß sich an sie noch ein kleiner, fensterloser, dunkler Raum (maksura) anschließt. Auch er 


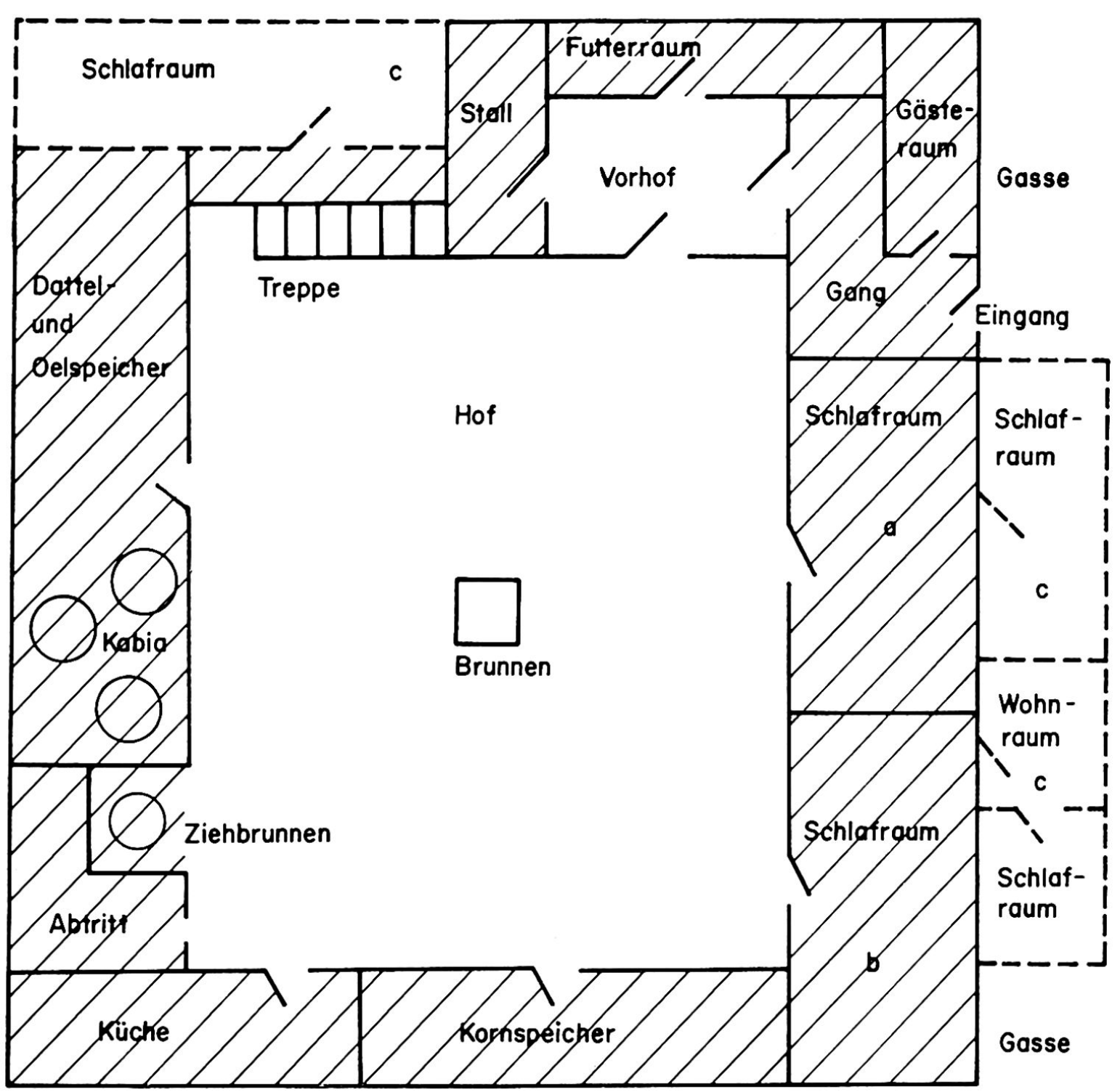

Abb. 4: Grundriß des Gehöftes der Familie Abdelkader ben Lagdar ben Sassi im Quartier Guitna in Tozeur. $a=$ Kinderschlafraum, $b=$ Elternschlafraum, $c=$ Schlafräume der Sohnsfamilien, alle drei (in der Zeichnung nicht schraffiert) im ersten Stock gelegen

dient zum Schlafen, doch nur zu ganz bestimmten Zeiten, nämlich während der kältesten Wintermonate und während des heißen Sommers über die Mittagszeit, im ersten Fall seiner relativen Wärme, im zweiten Fall seiner relativen Kühle wegen. Im Sommer schlafen die Leute nachts im Hof, entweder in Betten, die sie dort aufstellen, oder auf einem aus Bambusrohr, Latten oder Brettern über dem Erdboden errichteten und durch Steine gestützten Rost (sedda), auf den sie Teppiche legen. In den vornehmeren Häusern ist ein besonderer Eß/Wohnraum vorhanden.

In keinem Gehöft fehlen Küche (sabat), Speicher (dar el khsin oder khasin) und Latrine (duiria). Je nach dem Stand des Hausfriedens wird gemeinsame Küche oder Einzelküche - jede Sohn:familie kocht dann für sich - geführt. Im Speicher werden Korn, dieses in Körben, und Datteln und Olivenöl, diese beiden Lebensmittel in Gipsund Tongefäßen (kabia), aufbewahrt. Diese Gefäße sind meist groß, nicht selten fast mannshoch und vermögen bis $600 \mathrm{~kg}$ Datteln zu fassen. Im Herbst werden sie mit frischen Datteln gefüllt, die man mit den nackten Füßen feststampft. Der süße Saft, der sogenannte Dattelhonig, der sich während der ersten Wochen täglich infolge des Eigen- 
gewichts der Dattelmasse bildet, kann durch ein in Bodennähe angebrachtes Loch ausfließen und in einem Napf aufgefangen werden. Die großen Kabia wurden im Speicher drin hergestellt und in seinem Boden fest verankert. - Die Latrine ist nichts anderes als eine Grube mit zwei darüber gelegten Palmbalken. Sie liegt im Hause gewöhnlich längs der an die Gasse angrenzenden Seite. So kann sie von dieser aus durch ein Loch, das man an der betreffenden Stelle in die Hauswand schlägt, entleert werden.

Das Haus weist auch einen Stall (roa, rua) für die paar Ziegen und den Esel auf. Dieser liegt oft in der Nähe des Ganges. In den vornehmeren Häusern dient überdies ein besonderer Duschenraum der Körperpflege. Diesem wird das Wasser zugeführt, das ein im Hof sich befindender Ziehbrunnen liefert oder auch ein gewöhnlicher Brunnen, der vom Wasseramt gespeist wird.

Viele Gehöfte sind zweigeschoßig. Die Räume des Obergeschoßes, im Djerid ganz allgemein als Ghorfas bezeichnet, finden als Schlafzimmer, häufiger aber, sind sie doch stärker als die des Erdgeschoßes dem Luftzug ausgesetzt, als Speicher Verwendung. Die Luft kann durch eine Anzahl Mauerschlitze in sie eintreten. An Stangen, die quer von Wand zu Wand ziehen, werden darin Datteltrauben zum Trocknen aufgehängt. Das Obergeschoß ist nicht immer voll, das heißt um den ganzen Hof herum, ausgebaut; es besteht bisweilen nur aus einem einzigen, direkt über der Gasse gelegenen Raum. Wo sich ein paar solche zu verschiedenen Häusern gehörende Räume hintereinander folgen, entstand eine Tunnelgasse (portal). Dieses Hinausbauen ist seit wenigen Jahren verboten. Manchmal weist das Obergeschoß, zu dem vom Hof aus eine Steintreppe (druj) führt, auch noch einen kurzen Arkadengang auf.

Der Bau eines größeren Gehöftes dauert bei einem Einsatz von drei oder vier Maurern und sechs bis acht Handlangern ungefähr vier Monate. Während der Bauzeit laden die künftigen Hausinsaßen die Arbeiter zu festlichen Mahlzeiten ein, so beim Beginn des Baus - ein Hahn oder Huhn wird auf der Baustelle getötet -, nach dem Anbringen der Tür- und Fenstersturze - diese werden mit dem Blut eines getöteten Hahns oder Huhns beschmiert - und beim Bezug des Hauses; dann muß gewöhnlich ein Schaf sein Leben lassen.

\section{LA MAISON DU DJERID (SUD-TUNISIE)}

Un des traits caractéristiques principaux du Bled el Djerid, région située à l'ouest du Chott el Djerid, est la manière de construire. Les maisons sont érigées en briques fabriquées sur place, spécialement dans les oasis les plus importantes comme Tozeur et Nefta. Les habitants ont su disposer cette pierre sur les façades en de jolis ornements géométriques. Il est question aussi de la construction des murs et du toit et de la disposition de l'intérieur de la maison en chambres d'habitation, cuisine, grenier, etc. Par des chambres construites au-dessus des ruelles et appartenant à des maisons voisines les ruelles ont été transformées en tunnels.

\section{DER GLETSCHER AM ERCIYAS DAGH UND DAS PROBLEM DER REZENTEN SCHNEEGRENZE IM ANATOLISCHEN UND MEDITERRANEN RAUM}

\section{BRUNO MESSERLI}

\section{DER GLETSCHER AM ERCIYAS DAGH}

1.1. Sein früherer Stand. Die älteste Beschreibung des antiken Argaeus überliefert uns der griechische Geograph Strabo am Ende des 1. Jahrhunderts v. Chr. Er schildert die Aussicht vom schneebedeckten Gipfel, den zu betreten nur wenigen Menschen vergönnt gewesen sei. (lib. XII 2 in 36, S. 129). Nach dieser ersten Notiz fehlt uns jede Nachricht bis zu den Forschungsreisen des 19. Jahrhunderts. 